that were recommended to them. Although $77 \%$ were aware of a form of recommended vaccine, 39\% were not receiving them. Wider education of our IBD patients as well as their primary care doctors should be implemented to increase awareness and uptake of vaccines to provide adequate protection to this vulnerable group.

Disclosure of Interest None Declared.

\section{PWE-102 THE USE OF TPMT ENZYME TESTING IN MANAGING THIOPURINE THERAPY}

doi:10.1136/gutjnl-2013-304907.390

1."M Widlak, 'M Smith, '1 Slater, 'L Wood, 'S De Silva. 'Gastroenterology, Russells Hall Hospital, The Dudley Group NHS Foundation Trust, Dudley, UK

Introduction Thiopurine methyl transferase (TPMT) enzyme deficiency is associated with azathioprine-induced myelosuppression and hepatotoxicity. We aimed to assess thiopurine methyl transferase levels (TPMT) in patients with inflammatory bowel disease (IBD) and to determine how these levels impact on thiopurine dosing and further treatment.

Methods Retrospective analysis of all adult IBD patients treated with azathioprine (AZA) who had TPMT levels checked in our department 2008 - 2012. Patient demographics, AZA dose, blood parameters, adverse effects and reason for discontinuation were recorded. Patients were treated to try and achieve a target dose of $2.0-2.5 \mathrm{mg} / \mathrm{kg}$ of AZA. Our laboratory enzyme assay defined normal TPMT level as 68-150 mU/L.

Results A cohort of 135 patients receiving azathioprine were identified. Mean age was 43 years (SD 17), M:F ratio 1:1. 72 patients $(53 \%)$ had Crohn's disease, 60 (44\%) ulcerative colitis and $3(3 \%)$ indeterminate colitis. Mean starting dose of AZA $1.7 \mathrm{mg} / \mathrm{kg}$ (SD $0.6)$, maintenance dose $1.8 \mathrm{mg} / \mathrm{kg}$ (SD 0.5). 63 (47\%) were initially dosed with $\geq 2.0 \mathrm{mg} / \mathrm{kg}$. The mean TPMT level was $100 \mathrm{mU} / \mathrm{L}$ (SD 23); 14 patients (10\%) had low levels. Patients with low TPMT levels were generally started at lower doses of AZA $1.3 \mathrm{mg} / \mathrm{kg}$ (SD $0.7)$ vs $1.8 \mathrm{mg} / \mathrm{kg}$ (SD 0.6).

Mean length of follow up on AZA was 10.6 months (range 0.1 44). 50 out of 135 patients ( $37 \%$ ) exhibited adverse effects during treatment (representing 44 of the 121 (36\%) with normal TPMT levels, 6 of 14 (43\%) with low TPMT). Median time to adverse effects was 3 months (range 0.1-22). Leucopenia developed in 11 $(22 \%)$, deranged liver biochemistry 24 (48\%), adverse symptoms 24 (48\%). There was one case of pancreatitis and lymphoma respectively. Adverse effects were managed by discontinuation in $30(60 \%)$, dose reduction 14 (28\%), monitoring only $6(12 \%)$.

In the 6 patients with low TPMT levels, 4 developed deranged liver biochemistry and 2 had leucopenia; in 50\% AZA was discontinued. The median time to adverse effects was 11 months (range $2-22$ ), with 5 out of 6 patients developing adverse effects after more than 6 months of treatment.

Conclusion In our cohort low TPMT enzyme activity was not associated with an early onset of adverse effects or an excess of adverse effects when compared to the normal TPMT group. We would suggest that the value of TPMT level testing prior to thiopurine therapy may be less of a priority than vigilant monitoring of blood parameters during AZA therapy in identifying serious adverse effects.

Disclosure of Interest None Declared.

\section{PWE-103 VITAMIN D DEFICIENCY IN CROHNS DISEASE AND ULCERATIVE COLITIS}

doi:10.1136/gutjnl-2013-304907.391

1."M S Mohammad, 'J Mayberry. 'Gastroenterology, University Hospitals of Leicester, Leicester, UK
Introduction The role of vitamin $\mathrm{D}$ in inflammation and its possible cancer protective effects are now coming to the fore. Inflammatory bowel disease (IBD) provides a model where both roles can be assessed. Early studies have indicated that vitamin $\mathrm{D}$ deficiency is common amongst patients with this condition. Leicester in the UK has a significant population of patients from both English and South Asian backgrounds and so allows comparisons to be made between people of different ethnicities and different experiences of vitamin $\mathrm{D}$ deficiency.

Methods Vitamin D (25 OH D) status was measured in 148 consecutive patients with IBD, who attended the gastroenterology clinics during a period of one year (Jan to Dec 2011). Vitamin D levels were graded as severe deficiency $(<15 \mathrm{nmol} / \mathrm{l})$, moderate deficiency $(16-30 \mathrm{nmol} / \mathrm{l})$, insufficiency $(31-50 \mathrm{nmol} / \mathrm{l})$, adequate $(51-75$ $\mathrm{nmol} / \mathrm{l})$, and ideal (> $76 \mathrm{nmol} / \mathrm{l})$. All case notes were reviewed and data analysed using $\chi^{2}$ statistic.

Results 148 patients had IBD, 64\% were women and 36\% men. 93 patients (61\%) with ulcerative colitis (UC) and 55 (39\%) Crohns disease (CD). Of the 93 patients with UC, 50 were English and 43 South Asian. Of the 55 patients with CD, 43 were English and 12 of South Asian background. The proportions amongst both communities and across the disease groups were not significantly different when analysed with a $\chi^{2}$ statistics.

Abstract PWE-103 Table Vitamin D Levels in patients with inflammatory bowel disease in Leicester

\begin{tabular}{lllll}
\hline Ulcerative colitis & $<\mathbf{1 5 n m} / \mathbf{l t}$ & $\mathbf{1 5 - 3 0 n m / l t}$ & $\mathbf{3 0 - 5 0 n m / l t}$ & $>\mathbf{5 0 n m} / \mathbf{t t}$ \\
\hline English (50 pts) & $3(6 \%)$ & $17(34 \%)$ & $12(24 \%)$ & $18(36 \%)$ \\
Asian (43 pts) & $16(37 \%)$ & $13(31 \%)$ & $12(28 \%)$ & $12(28 \%)$ \\
Crohns disease & & & & \\
English (42 pts) & $7(6 \%)$ & $14(34 \%)$ & $8(19 \%)$ & $13(31 \%)$ \\
Asian (12 pts) & $2(37 \%)$ & $4(31 \%)$ & $3(25 \%)$ & $3(25 \%)$ \\
\hline
\end{tabular}

Conclusion Vitamin D deficiency is common in all patients with inflammatory bowel disease. It plays a vital role in bone health, immune regulation and cancer prevention in IBD. The optimal target level of $25(\mathrm{OH})$ vitamin $\mathrm{D}$ in IBD patients is uncertain as is the best therapeutic modality. The precise threshold for $25(\mathrm{OH})$ vitamin $\mathrm{D}$ level is poorly defined in the literature and so is the dosing and duration of treatment. Its frequency is almost similar in South Asian and English patients with Crohn's disease which suggests that mal-absorption of the vitamin may play a part in the vitamin $\mathrm{D}$ deficiency. In contrast it is commoner in South Asian patients with ulcerative colitis than amongst English patients and this warrants further investigation. It may suggest that in the future South Asian patients will be at greater risk of colonic carcinoma as a complication of ulcerative colitis.

Disclosure of Interest None Declared.

\section{PWE-104 VITAMIN D IN INFLAMMATORY BOWEL DISEASE. A SEMI-QUALITATIVE ASSESSMENT OF THE PATIENT EXPERIENCE}

doi:10.1136/gutjnl-2013-304907.392

1." M S Mohammad, 'J Mayberry. 'Gastroenterology, University Hospitals of Leicester, Leicester, UK

Introduction Vitamin D plays a vital role in bone health, immune regulation and cancer prevention in inflammatory bowel disease (IBD). Our understanding has increased remarkably in the past decade, although the mechanism of its influence in IBD 